\title{
The Positive Properties of Green's Function for Fractional Differential Equations and Its Applications
}

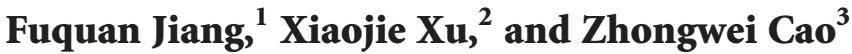 \\ ${ }^{1}$ The Department of Foundation, Harbin Finance University, Harbin 150030, China \\ ${ }^{2}$ College of Science, China University of Petroleum (East China), Qingdao 266580, China \\ ${ }^{3}$ Department of Applied Mathematics, Changchun Taxation College, Changchun 130117, China
}

Correspondence should be addressed to Fuquan Jiang; jfqsz@163.com

Received 13 July 2012; Revised 10 December 2012; Accepted 24 December 2012

Academic Editor: Dumitru Baleanu

Copyright (C) 2013 Fuquan Jiang et al. This is an open access article distributed under the Creative Commons Attribution License, which permits unrestricted use, distribution, and reproduction in any medium, provided the original work is properly cited.

We consider the properties of Green's function for the nonlinear fractional differential equation boundary value problem: $\mathbf{D}_{0+}^{\alpha} u(t)+$ $f(t, u(t))+e(t)=0,0<t<1, u(0)=u^{\prime}(0)=\cdots=u^{(n-2)}(0)=0, u(1)=\beta u(\eta)$, where $n-1<\alpha \leq n, n \geq 3,0<\beta \leq 1,0 \leq \eta \leq 1, \mathbf{D}_{0+}^{\alpha}$ is the standard Riemann-Liouville derivative. Here our nonlinearity $f$ may be singular at $u=0$. As applications of Green's function, we give some multiple positive solutions for singular boundary value problems by means of Schauder fixed-point theorem.

\section{Introduction}

Fractional differential equations have been of great interest recently. This is due to the intensive development of the theory of fractional calculus itself as well as its applications. Apart from diverse areas of mathematics, fractional differential equations arise in rheology, dynamical processes in selfsimilar and porous structures, fluid flows, electrical networks, viscoelasticity, chemical physics, and many other branches of science. For details, see [1-10].

It should be noted that most of the papers and books on fractional calculus are devoted to the solvability of linear initial fractional differential equations on terms of special functions. Recently, there are some papers dealing with the existence and multiplicity of solution to the nonlinear fractional differential equations boundary value problems, see [11-17].

Bai [14] investigated the existence and uniqueness of positive solutions for a nonlocal boundary value problem of fractional differential equation

$$
\begin{gathered}
\mathbf{D}_{0+}^{\alpha} u(t)+f(t, u(t))=0, \quad 0<t<1, \\
u(0)=0, \quad u(1)=\beta u(\eta),
\end{gathered}
$$

by contraction map principle and fixed-point index theory, where $1<\alpha \leq 2,0<\beta \eta^{\alpha-1}<1,0<\eta<1, \mathbf{D}_{0+}^{\alpha}$ is the standard Riemann-Liouville derivative. The function $f$ is continuous on $[0,1] \times[0, \infty)$.

Li et al. [17] investigated the the existence and multiplicity results of positive solutions for the nonlinear differential equation of fractional order

$$
\begin{aligned}
& \mathbf{D}_{0+}^{\alpha} u(t)+f(t, u(t))=0, \quad 0<t<1, \\
& u(0)=0, \quad \mathbf{D}_{0+}^{\beta} u(1)=a \mathbf{D}_{0+}^{\beta} u(\xi),
\end{aligned}
$$

by using some fixed-point theorems, where $1<\alpha \leq 2,0<$ $\beta \leq 1,0 \leq a \leq 1, \xi \in(0,1), a \xi^{\alpha-\beta-2} \leq 1-\beta, 0 \leq \alpha-\beta-1$, $\mathrm{D}_{0+}^{\alpha}$ is the standard Riemann-Liouville derivative.

$\mathrm{Xu}$ and Fei [18] considered the properties of Green's function for the nonlinear fractional differential equation boundary value problem

$$
\begin{gathered}
\mathbf{D}_{0+}^{\alpha} u(t)+f(t, u(t))+e(t)=0, \quad 0<t<1, \\
u(0)=0, \quad \mathbf{D}_{0+}^{\beta} u(1)=a \mathbf{D}_{0+}^{\beta} u(\xi),
\end{gathered}
$$

where $1<\alpha \leq 2,0<\beta \leq 1,0 \leq a \leq 1,0<\xi<1$, $\alpha-\beta-1 \geq 0, \mathbf{D}_{0+}^{\alpha}$ is the standard Riemann-Liouville derivative. Here the nonlinearity $f$ may be singular at $u=0$. 
As applications of Green's function, they give some existence of positive solutions for singular boundary value problems by means of Schauder fixed-point theorem. Here they consider the case: $\gamma_{*}=0, \gamma_{*} \geq 0, \gamma^{*} \leq 0$.

In this paper, we consider the singular boundary value problem

$$
\begin{array}{cl}
D_{0+}^{\alpha} u(t)+f(t, u(t))+e(t)=0, & 0<t<1, \\
u(0)=u^{\prime}(0)=\cdots=u^{(n-2)}(0)=0, & u(1)=\beta u(\eta),
\end{array}
$$

where $n-1<\alpha \leq n, n \geq 3,0<\beta, \eta<1$ is a real constant, $D_{0+}^{\alpha}$ is the standard Riemann-Liouville fractional derivative. We will deduce a property of Green's function. The result we establish in Section 2 can be stated as follow.

Theorem 1. The Function $G(t, s)$ defined by (12) is continuous and satisfies

$$
\begin{array}{r}
\frac{M t^{\alpha-1} s(1-s)^{\alpha-1}}{\Gamma(\alpha)\left(1-\beta \eta^{\alpha-1}\right)} \leq G(t, s) \leq \frac{t^{\alpha-1}(1-s)^{\alpha-1}}{\Gamma(\alpha)\left(1-\beta \eta^{\alpha-1}\right)} \\
\text { for } t, s \in[0,1]
\end{array}
$$

where $0<M=\min \left\{1-\beta \eta^{\alpha-1}, \beta \eta^{\alpha-2}(1-\eta), \beta \eta^{\alpha-1}\right\}<1$.

In this paper, we give some existence of positive solutions for singular boundary value problems by means of Schauder fixed-point theorem for the case: $\gamma_{*}=0, \gamma_{*} \geq 0, \gamma^{*} \leq 0$, $\gamma_{*}<0<\gamma^{*}$.

The paper is organized as follows. In Section 2, we state some known results and give a property of Green's function. In Section 3, using Schauder fixed-point theorem, the existence of positive solutions to singular problems are obtained.

\section{Background Materials}

For the convenience of the reader, we present here the necessary definitions from fractional calculus theory.

Definition 2 (see [7]). The Riemann-Liouville fractional integral of order $\alpha>0$ of a function $y:(0, \infty) \rightarrow R$ is given by

$$
I_{0+}^{\alpha} y(t)=\frac{1}{\Gamma(\alpha)} \int_{0}^{t}(t-s)^{\alpha-1} y(s) d s,
$$

provided the right side is pointwise defined on $(0, \infty)$.

Definition 3 (see [7]). The Riemann-Liouville fractional derivative of order $\alpha>0$ of a continuous function $y:(0, \infty) \rightarrow$ $R$ is given by

$$
D_{0+}^{\alpha} y(t)=\frac{1}{\Gamma(n-\alpha)}\left(\frac{d}{d t}\right)^{n} \int_{0}^{t} \frac{y(s)}{(t-s)^{\alpha-n+1}} d s,
$$

where $n=[\alpha]+1,[\alpha]$ denotes the integer part of number $\alpha$, provided that the right side is pointwise defined on $(0, \infty)$.
From the definition of Riemann-Liouville's derivative, we can obtain the statement.

Lemma 4 (see [7]). Let $\alpha>0$, if one assumes that $u \in C(0,1) \cap$ $L(0,1)$, then the fractional differential equation

$$
\mathbf{D}_{0+}^{\alpha} u(t)=0
$$

has $u(t)=C_{1} t^{\alpha-1}+C_{2} t^{\alpha-2}+\cdots+C_{N} t^{\alpha-N}, C_{i} \in R, i=$ $1,2, \ldots, N$, as unique solutions, where $N$ is the smallest integer greater than or equal to $\alpha$.

Lemma 5 (see [7]). Assume that $u \in C(0,1) \cap L(0,1)$ with a fractional derivative of order $\alpha>0$ that belongs to $C(0,1) \cap$ $L(0,1)$. Then,

$$
I_{0+}^{\alpha} \mathbf{D}_{0+}^{\alpha} u(t)=u(t)+C_{1} t^{\alpha-1}+C_{2} t^{\alpha-2}+\cdots+C_{N} t^{\alpha-N},
$$

for some $C_{i} \in R, i=1,2, \ldots, N, N$ is the smallest integer greater than or equal to $\alpha$.

Lemma 6. Given $h \in C(0,1)$ the problem

$$
\begin{gathered}
D_{0+}^{\alpha} u(t)+h(t)=0, \quad 0<t<1, n-1<\alpha \leq n, n \geq 3 \\
u(0)=u^{\prime}(0)=\cdots=u^{(n-2)}(0)=0 \\
u(1)=\beta u(\eta), \quad 0<\beta, \eta<1
\end{gathered}
$$

is equivalent to

$$
u(t)=\int_{0}^{1} G(t, s) h(s) d s
$$

where

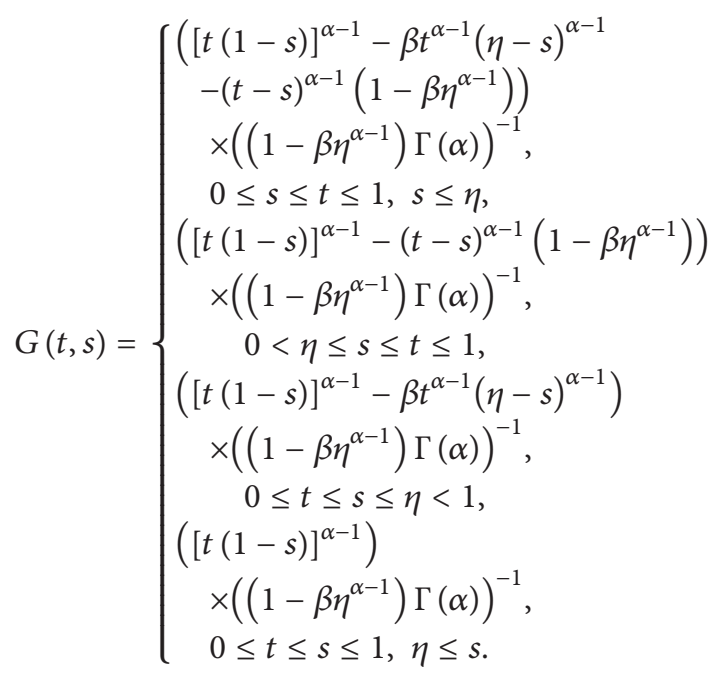

Proof. We can apply Lemma 5 to reduce (10) to an equivalent integral equation

$$
u(t)=-I_{0+}^{\alpha} h(t)+C_{1} t^{\alpha-1}+C_{2} t^{\alpha-2}+\cdots+C_{n} t^{\alpha-n},
$$


for some $C_{1}, C_{2}, \ldots, C_{n} \in R$. Consequently, the general solution of (10) is

$$
\begin{aligned}
u(t)= & -\frac{1}{\Gamma(\alpha)} \int_{0}^{t}(t-s)^{\alpha-1} h(s) d s \\
& +C_{1} t^{\alpha-1}+C_{2} t^{\alpha-2}+\cdots+C_{n} t^{\alpha-n} .
\end{aligned}
$$

$\operatorname{By} u(0)=u^{\prime}(0)=\cdots=u^{(n-2)}(0)=0$, this is $C_{2}=C_{3}=\cdots=$ $C_{n}=0$.

On the other hand, $u(1)=\beta u(\eta)$ combining with

$$
\begin{gathered}
u(1)=-\frac{1}{\Gamma(\alpha)} \int_{0}^{1}(1-s)^{\alpha-1} h(s) d s+C_{1}, \\
u(\eta)=-\frac{1}{\Gamma(\alpha)} \int_{0}^{\eta}(\eta-s)^{\alpha-1} h(s) d s+C_{1} \eta^{\alpha-1}
\end{gathered}
$$

yields

$$
\begin{aligned}
C_{1}= & \frac{1}{1-\beta \eta^{\alpha-1}} \int_{0}^{1} \frac{(1-s)^{\alpha-1}}{\Gamma(\alpha)} h(s) d s \\
& -\frac{\beta}{1-\beta \eta^{\alpha-1}} \int_{0}^{\eta} \frac{(\eta-s)^{\alpha-1}}{\Gamma(\alpha)} h(s) d s .
\end{aligned}
$$

Therefor, the unique solution of problem (10) is

$$
\begin{aligned}
u(t)= & -\int_{0}^{t} \frac{(t-s)^{\alpha-1}}{\Gamma(\alpha)} h(s) d s \\
& +\frac{1}{1-\beta \eta^{\alpha-1}} \int_{0}^{1} \frac{(t(1-s))^{\alpha-1}}{\Gamma(\alpha)} h(s) d s \\
& -\frac{\beta}{1-\beta \eta^{\alpha-1}} \int_{0}^{\eta} \frac{(t(\eta-s))^{\alpha-1}}{\Gamma(\alpha)} h(s) d s
\end{aligned}
$$

For $t \leq \eta$, we have

$$
\begin{aligned}
u(t)= & -\int_{0}^{t} \frac{(t-s)^{\alpha-1}}{\Gamma(\alpha)} h(s) d s \\
& +\frac{1}{1-\beta \eta^{\alpha-1}}\left[\left(\int_{0}^{t}+\int_{t}^{\eta}+\int_{\eta}^{1}\right) \frac{(t(1-s))^{\alpha-1}}{\Gamma(\alpha)} h(s) d s\right] \\
& -\frac{\beta}{1-\beta \eta^{\alpha-1}}\left[\left(\int_{0}^{t}+\int_{t}^{\eta}\right) \frac{(t(\eta-s))^{\alpha-1}}{\Gamma(\alpha)} h(s) d s\right] \\
= & \int_{0}^{t}\left(\left([t(1-s)]^{\alpha-1}-\beta[t(\eta-s)]^{\alpha-1}\right.\right. \\
& \left.-(t-s)^{\alpha-1}\left(1-\beta \eta^{\alpha-1}\right)\right) \\
& \left.\times\left(\left(1-\beta \eta^{\alpha-1}\right) \Gamma(\alpha)\right)^{-1}\right) h(s) d s
\end{aligned}
$$

$$
\begin{aligned}
& +\int_{t}^{\eta} \frac{[t(1-s)]^{\alpha-1}-\beta[t(\eta-s)]^{\alpha-1}}{\left(1-\beta \eta^{\alpha-1}\right) \Gamma(\alpha)} h(s) d s \\
& +\int_{\eta}^{1} \frac{[t(1-s)]^{\alpha-1}}{\left(1-\beta \eta^{\alpha-1}\right) \Gamma(\alpha)} h(s) d s \\
& =\int_{0}^{1} G(t, s) h(s) d s .
\end{aligned}
$$

For $t \geq \eta$, we have

$$
\begin{aligned}
& u(t)=-\left[\left(\int_{0}^{\eta}+\int_{\eta}^{t}\right) \frac{(t-s)^{\alpha-1}}{\Gamma(\alpha)} h(s) d s\right] \\
&-\frac{\beta}{1-\beta \eta^{\alpha-1}} \int_{0}^{\eta} \frac{(t(\eta-s))^{\alpha-1}}{\Gamma(\alpha)} h(s) d s \\
&+\frac{1}{1-\beta \eta^{\alpha-1}}\left[\left(\int_{0}^{\eta}+\int_{\eta}^{t}+\int_{t}^{1}\right) \frac{(t(1-s))^{\alpha-1}}{\Gamma(\alpha)} h(s) d s\right] \\
&= \int_{0}^{\eta}\left((t(1-s)]^{\alpha-1}-\beta[t(\eta-s)]^{\alpha-1}\right. \\
&+\int_{\eta}^{t} \frac{\left.-(t-s)^{\alpha-1}\left(1-\beta \eta^{\alpha-1}\right)\right)}{\left.\times\left(\left(1-\beta \eta^{\alpha-1}\right) \Gamma(\alpha)\right)^{-1}\right) h(s) d s} \\
&+\int_{t}^{1} \frac{[t(1-s)]^{\alpha-1}}{\left(1-\beta \eta^{\alpha-1}\right) \Gamma(\alpha)} h(s) d s \\
&= \int_{0}^{1} G(t, s) h(s) d s . \\
&\left(1-\beta \eta^{\alpha-1}\right) \Gamma(\alpha) \\
&
\end{aligned}
$$

The proof is complete.

Proof of Theorem 1. It is easy to prove that $G(t, s)$ is continuous on $[0,1] \times[0,1]$, here we omit it. In the following, we consider $\left(1-\beta \eta^{\alpha-1}\right) \Gamma(\alpha) G(t, s)$. When $0 \leq s<t \leq 1, s<\eta$, let

$$
\begin{aligned}
g_{1}(t)= & {[t(1-s)]^{\alpha-1}-\beta t^{\alpha-1}(\eta-s)^{\alpha-1} } \\
& -(t-s)^{\alpha-1}\left(1-\beta \eta^{\alpha-1}\right) .
\end{aligned}
$$

We have

$$
\begin{aligned}
g_{1}(t)= & {[t(1-s)]^{\alpha-1}-\beta t^{\alpha-1}(\eta-s)^{\alpha-1} } \\
& -(t-s)^{\alpha-1}\left(1-\beta \eta^{\alpha-1}\right) \\
= & {[t(1-s)]^{\alpha-1}-\beta \eta^{\alpha-1} t^{\alpha-1}\left(1-\frac{s}{\eta}\right)^{\alpha-1} } \\
& -t^{\alpha-1}\left(1-\frac{s}{t}\right)^{\alpha-1}\left(1-\beta \eta^{\alpha-1}\right)
\end{aligned}
$$




$$
\begin{aligned}
\geq & {[t(1-s)]^{\alpha-1}-\beta \eta^{\alpha-1} t^{\alpha-1}\left(1-\frac{s}{\eta}\right)^{\alpha-1} } \\
& -t^{\alpha-1}(1-s)^{\alpha-1}\left(1-\beta \eta^{\alpha-1}\right) \\
= & t^{\alpha-1} \beta \eta^{\alpha-1}\left[(1-s)^{\alpha-1}-\left(1-\frac{s}{\eta}\right)^{\alpha-1}\right] \\
= & t^{\alpha-1} \beta \eta^{\alpha-1}\left[(1-s)^{\alpha-1}-\left(1-\frac{s}{\eta}\right)^{\alpha-2}\left(1-\frac{s}{\eta}\right)\right] \\
\geq & t^{\alpha-1} \beta \eta^{\alpha-1}(1-s)^{\alpha-2}\left[1-s-\left(1-\frac{s}{\eta}\right)\right] \\
= & t^{\alpha-1} \beta \eta^{\alpha-1}(1-s)^{\alpha-2} s \frac{1-\eta}{\eta} \\
\geq & \beta \eta^{\alpha-1} t^{\alpha-1}(1-s)^{\alpha-1} s \frac{1-\eta}{\eta} \\
\geq & M t^{\alpha-1}(1-s)^{\alpha-1} s .
\end{aligned}
$$

When $0<\eta \leq s<t \leq 1$, let

$$
g_{2}(t)=[t(1-s)]^{\alpha-1}-(t-s)^{\alpha-1}\left(1-\beta \eta^{\alpha-1}\right) .
$$

We have

$$
\begin{aligned}
g_{2}(t) & =[t(1-s)]^{\alpha-1}-(t-s)^{\alpha-1}\left(1-\beta \eta^{\alpha-1}\right) \\
& =[t(1-s)]^{\alpha-1}-t^{\alpha-1}\left(1-\frac{s}{t}\right)^{\alpha-1}\left(1-\beta \eta^{\alpha-1}\right) \\
& \geq[t(1-s)]^{\alpha-1}-t^{\alpha-1}(1-s)^{\alpha-1}\left(1-\beta \eta^{\alpha-1}\right) \\
& =\beta \eta^{\alpha-1}(t-t s)^{\alpha-1} \\
& \geq M t^{\alpha-1}(1-s)^{\alpha-1} s .
\end{aligned}
$$

When $0 \leq t \leq s \leq \eta<1$, let $g_{3}(t)=[t(1-s)]^{\alpha-1}-\beta t^{\alpha-1}(\eta-$ $s)^{\alpha-1}$, we have

$$
\begin{aligned}
g_{3}(t) & =[t(1-s)]^{\alpha-1}-\beta t^{\alpha-1}(\eta-s)^{\alpha-1} \\
& =[t(1-s)]^{\alpha-1}-\beta \eta^{\alpha-1} t^{\alpha-1}\left(1-\frac{s}{\eta}\right)^{\alpha-1} \\
& \geq[t(1-s)]^{\alpha-1}-\beta \eta^{\alpha-1} t^{\alpha-1}(1-s)^{\alpha-1} \\
& =\left(1-\beta \eta^{\alpha-1}\right) t^{\alpha-1}(1-s)^{\alpha-1} \\
& \geq M t^{\alpha-1}(1-s)^{\alpha-1} s .
\end{aligned}
$$

When $0 \leq t \leq s \leq 1, \eta \leq s$, we have

$$
\left(1-\beta \eta^{\alpha-1}\right) \Gamma(\alpha) G(t, s)=[t(1-s)]^{\alpha-1} \geq M t^{\alpha-1}(1-s)^{\alpha-1} s .
$$

It is easy to see that $G(t, s) \leq t^{\alpha-1}(1-s)^{\alpha-1} / \Gamma(\alpha)\left(1-\beta \eta^{\alpha-1}\right)$. Thus, the proof is complete.
Let us fix some notations to be used in the following. "For a.e." means "for almost every". Given $a \in L^{1}(0,1)$, we write $a>$ 0 if $a \geq 0$ for a.e. $t \in[0,1]$, and it is positive in a set of positive measure, we write $f \in \operatorname{Car}((0,1) \times(0,+\infty),(0,+\infty))$ if $f$ : $(0,1) \times(0,+\infty) \rightarrow(0,+\infty)$ is a $L^{1}$-caratheodory function, that is, the map $x \mapsto f(t, x)$ is continuous for a.e. $t \in(0,1)$, and the map $t \mapsto f(t, x)$ is measurable for all $x \in(0,+\infty)$.

Let us define

$$
\begin{aligned}
& \gamma^{*}=\sup _{t \in[0,1]} \int_{0}^{1} \frac{G(t, s)}{t^{\alpha-1}} e(s) d s, \\
& \gamma_{*}=\inf _{t \in[0,1]} \int_{0}^{1} \frac{G(t, s)}{t^{\alpha-1}} e(s) d s .
\end{aligned}
$$

Then,

$$
t^{\alpha-1} \gamma_{*} \leq \int_{0}^{1} G(t, s) e(s) d s \leq t^{\alpha-1} \gamma^{*}
$$

\section{Main Results}

In this section, we establish the existence of positive solutions for equation

$$
\begin{array}{cl}
\mathbf{D}_{0+}^{\alpha} u(t)+f(t, u(t))+e(t)=0, & 0<t<1, \\
u(0)=u^{\prime}(0)=\cdots=u^{(n-2)}(0)=0, & u(1)=\beta u(\eta),
\end{array}
$$

where $n-1<\alpha \leq n, n \geq 3,0<\beta \leq 1,0 \leq \eta \leq 1$, $f \in \operatorname{Car}((0,1) \times(0,+\infty),(0,+\infty)), e(t) \in L^{1}[0,1], \mathbf{D}_{0+}^{\alpha}$ is the standard Riemann-Liouville derivative. The following is the first main result in this section.

Theorem 7. Suppose that the following conditions are satisfied.

$\left(\mathrm{H}_{1}\right)$ For each $L>0$, there exists a function $\phi_{L}>0$ such that $f\left(t, t^{\alpha-1} x\right) \geq \phi_{L}(t)$ for a.e. $t \in(0,1)$, all $x \in(0, L]$.

$\left(\mathrm{H}_{2}\right)$ There exist $g(x), h(x)$, and $k(t)>0$, such that

$$
\begin{aligned}
& 0 \leq f(t, x) \leq k(t)\{g(x)+h(x)\} \\
& \text { for a.e.t } \in(0,1), \text { all } x \in(0, \infty),
\end{aligned}
$$

here

$g:(0,+\infty)$

$\longrightarrow[0,+\infty)$ is continuous and nonincreasing,

$h:[0,+\infty)$

$\longrightarrow[0,+\infty)$ is continuous, and $\frac{h}{g}$ is nondecreasing. 
$\left(\mathrm{H}_{3}\right)$ There exist two positive constants $R>r>0$ such that

$$
\begin{gathered}
R>\Phi_{R 1}+\gamma_{*} \geq r>0, \\
\int_{0}^{1} k(s) g\left(r s^{\alpha-1}\right) d s<+\infty, \\
R \geq\left(1+\frac{h(R)}{g(R)}\right) \int_{0}^{1} \frac{(1-s)^{\alpha-1}}{\Gamma(\alpha)\left(1-\beta \eta^{\alpha-1}\right)} k(s) g\left(r s^{\alpha-1}\right) d s+\gamma^{*},
\end{gathered}
$$

and here

$$
\Phi_{R 1}=\int_{0}^{1} \frac{M s(1-s)^{\alpha-1}}{\Gamma(\alpha)\left(1-\beta \eta^{\alpha-1}\right)} \phi_{R}(s) d s
$$

Then, (28) has at least one positive solution.

Proof. Let $E=(C[0,1],\|\cdot\|)$, and $\Omega$ is a closed convex set defined as

$$
\Omega=\left\{x \in C[0,1]: t^{\alpha-1} r \leq x(t) \leq t^{\alpha-1} R \forall t \in[0,1]\right\},
$$

here $E=C[0,1]$ is the Banach space of continuous functions defined on $[0,1]$ with the norm

$$
\|x\|:=\max _{t \in[0,1]}|x(t)|
$$

and $R>r>0$ are positive constants to be given below.

Now, we define an operator $T: \Omega \rightarrow E$ by

$$
(T x)(t):=\int_{0}^{1} G(t, s)[f(s, x(s))+e(s)] d s .
$$

Then, (28) is equivalent to the fixed-point problem

$$
x=T x .
$$

Let $R$ be the positive constant satisfying $\left(\mathrm{H}_{3}\right)$ and

$$
\Phi_{R 1}+\gamma_{*} \geq r
$$

Then, we have $R>r>0$. Now, we prove $T(\Omega) \subset \Omega$. $\left(\mathrm{H}_{3}\right)$

In fact, for each $x \in \Omega$ and for all $t \in(0,1)$, by $\left(\mathrm{H}_{1}\right)$ and

$$
\begin{aligned}
(T x)(t) \geq & \int_{0}^{1} t^{\alpha-1} \frac{M s(1-s)^{\alpha-1}}{\Gamma(\alpha)\left(1-\beta \eta^{\alpha-1}\right)} \phi_{R}(s) d s \\
& +\int_{0}^{1} G(t, s) e(s) d s \\
\geq & t^{\alpha-1}\left[\Phi_{R 1}+\gamma_{*}\right] \geq t^{\alpha-1} r .
\end{aligned}
$$

On the other hand, by conditions $\left(\mathrm{H}_{2}\right)$ and $\left(\mathrm{H}_{3}\right)$, we have

$(T x)(t)$

$$
\begin{aligned}
& \leq \int_{0}^{1} t^{\alpha-1} \frac{(1-s)^{\alpha-\beta-1}}{\Gamma(\alpha)\left(1-a \xi^{\alpha-\beta-1}\right)} k(s) \\
& \quad \times\left[g\left(s^{\alpha-1} \frac{x(s)}{s^{\alpha-1}}\right)+h\left(s^{\alpha-1} \frac{x(s)}{s^{\alpha-1}}\right)\right] d s \\
& \quad+\int_{0}^{1} G(t, s) e(s) d s
\end{aligned}
$$

$$
\begin{aligned}
\leq t^{\alpha-1}\left[\int_{0}^{1} \frac{(1-s)^{\alpha-1}}{\Gamma(\alpha)\left(1-\beta \eta^{\alpha-1}\right)} k(s) g\left(s^{\alpha-1} \frac{x(s)}{s^{\alpha-1}}\right)\right. \\
\left.\quad \times\left\{1+\frac{h\left(s^{\alpha-1}\left(x(s) / s^{\alpha-1}\right)\right)}{g\left(s^{\alpha-1}\left(x(s) / s^{\alpha-1}\right)\right)}\right\} d s+\gamma^{*}\right]
\end{aligned}
$$

$\leq t^{\alpha-1}$

$$
\times\left[\left(1+\frac{h(R)}{g(R)}\right) \int_{0}^{1} \frac{(1-s)^{\alpha-1}}{\Gamma(\alpha)\left(1-\beta \eta^{\alpha-1}\right)} k(s) g\left(r s^{\alpha-1}\right) d s+\gamma^{*}\right]
$$

$\leq t^{\alpha-1} R$

In conclusion, $T(\Omega) \subset \Omega$.

Finally, it is standard that $T: \Omega \rightarrow \Omega$ is a continuous and completely continuous operator. By a direct application of Schauder's fixed-point theorem, (28) has at least one positive solution $x(t) \in C[0,1]$, the proof is finished.

Case $1\left(\gamma_{*}=0\right)$. As an application of Theorem 7, we consider the case $\gamma_{*}=0$. The following corollary is a direct result of Theorem 7 with $r=\Phi_{R 1}$.

Corollary 8. Suppose that $f(t, x)$ satisfies conditions $\left(\mathrm{H}_{1}\right)$ $\left(\mathrm{H}_{2}\right)$. Furthermore, assume the following.

$\left(\mathrm{H}_{3}^{*}\right)$ There exists a positive constant $R>0$ such that

$$
\begin{gathered}
R>\Phi_{R 1}>0, \\
\int_{0}^{1} k(s) g\left[\left(\Phi_{R 1}\right) s^{\alpha-1}\right] d s<+\infty, \\
R \geq\left(1+\frac{h(R)}{g(R)}\right) \\
\times \int_{0}^{1} \frac{(1-s)^{\alpha-1}}{\Gamma(\alpha)\left(1-\beta \eta^{\alpha-1}\right)} k(s) g\left[\left(\Phi_{R 1}\right) s^{\alpha-1}\right] d s+\gamma^{*},
\end{gathered}
$$

and here

$$
\Phi_{R 1}=\int_{0}^{1} \frac{M s(1-s)^{\alpha-1}}{\Gamma(\alpha)\left(1-\beta \eta^{\alpha-1}\right)} \phi_{R}(s) d s .
$$

Then, (28) has at least one positive solution. 
From now on, let us define

$$
\begin{gathered}
\beta_{1}=\int_{0}^{1} \frac{M s^{1-\lambda(\alpha-1)}(1-s)^{\alpha-1}}{\Gamma(\alpha)\left(1-\beta \eta^{\alpha-1}\right)} k(s) d s, \\
\beta_{2}=\int_{0}^{1} \frac{s^{-\lambda(\alpha-1)}(1-s)^{\alpha-1}}{\Gamma(\alpha)\left(1-\beta \eta^{\alpha-1}\right)} k(s) d s .
\end{gathered}
$$

Example 9. Suppose that the nonlinearity in (28) is

$$
f(t, x)=\frac{k(t)}{x^{\lambda}}
$$

where $k>0,0<\lambda<1$ and

$$
\omega(\lambda):=\int_{0}^{1} k(s) s^{-\lambda(\alpha-1)} d s<+\infty .
$$

If $\gamma_{*}=0$, then (28) has at least one positive solution.

Proof. We will apply Corollary 8 . To this end, we take

$$
\phi_{L}(t)=\frac{k(t)}{\left(t^{\alpha-1} L\right)^{\lambda}}, \quad g(x)=\frac{1}{x^{\lambda}}, \quad h(x) \equiv 0,
$$

then $\left(\mathrm{H}_{1}\right)$ and $\left(\mathrm{H}_{2}\right)$ are satisfied since $\omega(\lambda)<+\infty$, and the existence condition $\left(\mathrm{H}_{3}^{*}\right)$ becomes

$$
\left(\frac{R^{\lambda}}{\beta_{1}}\right)^{\lambda} \beta_{2}+\gamma^{*} \leq R, \quad R>\frac{\beta_{1}}{R^{\lambda}}, \omega(\lambda)<+\infty,
$$

for some $R>0$. Since $0<\lambda<1$, we can choose $R>0$ large enough such that (46) is satisfied, and the proof is finished.

Example 10. Suppose that the nonlinearity in (28) is

$$
f(t, x)=k(s)\left(x^{-\lambda}+\mu x^{v}\right),
$$

where $0<\lambda<1, v \geq 0$ and $\mu \geq 0$ is a nonnegative parameter. For each $e(t)$ with $\gamma_{*}=0, \omega(\lambda)<+\infty$,

(i) if $\lambda+v<1-\lambda^{2}$, then (28) has at least one positive solution for each $\mu \geq 0$.

(ii) If $\lambda+v \geq 1-\lambda^{2}$, then (28) has at least one positive solution for each $0 \leq \mu<\mu_{1}$, where $\mu_{1}$ is some positive constant.

Proof. We will apply Corollary 8 . To this end, we take

$$
\phi_{L}(t)=\frac{k(t)}{\left(t^{\alpha-1} L\right)^{\lambda}}, \quad g(x)=x^{-\lambda}, \quad h(x)=\mu x^{\nu} .
$$

Then, $\left(\mathrm{H}_{1}\right)-\left(\mathrm{H}_{2}\right)$ are satisfied since $\omega(\lambda)<+\infty$. Now, the existence condition $\left(\mathrm{H}_{3}^{*}\right)$ becomes $\omega(\lambda)<+\infty$, and

$$
\mu \leq \frac{R^{1-\lambda^{2}} \beta_{1}^{\lambda}-\gamma^{*} \beta_{1}^{\lambda} R^{-\lambda^{2}}-\beta_{2}}{\beta_{2} R^{\lambda+v}},
$$

for some $R>0$ with $R^{1+\lambda}>\beta_{1}$. So, (28) has at least one positive solution for

$$
0<\mu<\mu_{1}=\sup _{R>\beta_{1}^{1 / 1+\lambda}} \frac{R^{1-\lambda^{2}} \beta_{1}^{\lambda}-\gamma^{*} \beta_{1}^{\lambda} R^{-\lambda^{2}}-\beta_{2}}{\beta_{2} R^{\lambda+v}} .
$$

Note that $\mu_{1}=\infty$ if $\lambda+v<1-\lambda^{2}$ and if $\lambda+v \geq 1-\lambda^{2}$, set

$$
l(R):=\frac{R^{1-\lambda^{2}} \beta_{1}^{\lambda}-\gamma^{*} \beta_{1}^{\lambda} R^{-\lambda^{2}}-\beta_{2}}{\beta_{2} R^{\lambda+v}}
$$

then, we have

$$
\begin{aligned}
l^{\prime}(R)=\frac{1}{R^{\lambda+v+1} \beta_{2}}[ & \left(1-\lambda^{2}-\lambda-v\right) R^{1-\lambda^{2}} \beta_{1}^{\lambda} \\
& \left.+\left(\lambda^{2}+\lambda+\nu\right) \gamma^{*} \beta_{1}^{\lambda} R^{-\lambda^{2}}+(\lambda+v) \beta_{2}\right] .
\end{aligned}
$$

Let the function $l(R)$ possess a maximum at $R_{0}$, then

$$
\begin{aligned}
\left(\lambda^{2}+\right. & \lambda+\nu-1) R_{0}^{1-\lambda^{2}} \beta_{1}^{\lambda} \\
& =\left(\lambda^{2}+\lambda+\nu \lambda\right) \gamma^{*} \beta_{1}^{\lambda} R_{0}^{-\lambda^{2}}+(\lambda+\nu) \beta_{2},
\end{aligned}
$$

so we have

$$
\left(\lambda+v-1+\lambda^{2}\right) \beta_{1}^{\lambda} R_{0} \geq(\lambda+v) \beta_{2} R_{0}^{\lambda^{2}},
$$

it is easy to find that $R_{0}>\left(\beta_{2} / \beta_{1}^{\lambda}\right)^{1 /\left(1-\lambda^{2}\right)}$ since $\lambda+\nu \geq 1-\lambda^{2}$, and $0<\lambda<1$. Finally, it would remain to prove $R_{0}>\beta_{1}^{1 / 1+\lambda}$. This is easily verified through elementary computations since $\beta_{1} \leq \beta_{2}$. We have the desired results (i) and (ii).

Case $2\left(\gamma_{*}>0\right)$. The next result explores the case when $\gamma_{*}>$ 0 . In this case $r=\gamma_{*}$.

Corollary 11. Suppose that $f(t, x)$ satisfies $\left(\mathrm{H}_{2}\right)$. Furthermore, assume the following.

$\left(\mathrm{H}_{4}\right)$ There exists $R>0, \int_{0}^{1} k(s) g\left(\gamma_{*} s^{\alpha-1}\right) d s<+\infty$, such that

$\left(1+\frac{h(R)}{g(R)}\right) \int_{0}^{1} \frac{(1-s)^{\alpha-1}}{\Gamma(\alpha)\left(1-\beta \eta^{\alpha-1}\right)} k(s) g\left(\gamma_{*} s^{\alpha-1}\right) d s+\gamma^{*} \leq R$.

If $\gamma_{*}>0$, then (28) has at least one positive solution.

Example 12. Suppose that the nonlinearity in (28) be (43) with $k>0, \lambda>0$. If $\gamma_{*}>0, \omega(\lambda)<+\infty$, then (28) has at least one positive solution.

Proof. We will apply Corollary 11. Take $k(t), g(x)$, and $h(x)$ as the same in the proof of Example 9. Then, $\left(\mathrm{H}_{2}\right)$ is satisfied, and the existence condition $\left(\mathrm{H}_{4}\right)$ is satisfied if we take $R>0$ with $R \geq \beta_{2} / \gamma_{*}^{\lambda}+\gamma^{*}$, and $\omega(\lambda)<+\infty$.

Example 13. Let the nonlinearity in (28) be (47) with $\lambda>0$ and $v \geq 0$. For each $e(t)$ with $\gamma_{*}>0, \omega(\lambda)<+\infty$, 
(i) if $\lambda+v<1$, then (28) has at least one positive solution for each $\mu \geq 0$.

(ii) If $\lambda+v \geq 1$, then (28) has at least one positive solution for each $0 \leq \mu<\mu_{2}$, where $\mu_{2}$ is some positive constant.

Proof. We will apply Corollary 11. To this end, we take $g(x)$, $h(x)$, and $k(t)$ as the same in the proof of Example 10, then $\left(\mathrm{H}_{2}\right)$ is satisfied, and the existence condition $\left(\mathrm{H}_{4}\right)$ becomes $\omega(\lambda)<+\infty$,

$$
\mu \leq \frac{R \gamma_{*}^{\lambda}-\gamma^{*} \gamma_{*}^{\lambda}-\beta_{2}}{\beta_{2} R^{\lambda+v}},
$$

for some $R>0$. So, (28) has at least one positive solution for

$$
0<\mu<\mu_{2}=\sup _{R>0} \frac{R \gamma_{*}^{\lambda}-\gamma^{*} \gamma_{*}^{\lambda}-\beta_{2}}{\beta_{2} R^{\lambda+v}} .
$$

Note that $\mu_{2}=\infty$ if $\lambda+v<1$ and $\mu_{2}=\gamma_{*}^{\lambda} / \beta_{2}$ if $\lambda+v=1$, and if $\lambda+v>1$ set

$$
l(R):=\frac{R \gamma_{*}^{\lambda}-\gamma^{*} \gamma_{*}^{\lambda}-\beta_{2}}{\beta_{2} R^{\lambda+v}} .
$$

The function $l(R)$ possesses a maximum at

$$
R_{0}:=\frac{(\lambda+v)\left(\gamma^{*} \gamma_{*}^{\lambda}+\beta_{2}\right)}{(\lambda+v-1) \gamma_{*}^{\lambda}},
$$

then $\mu_{2}=l\left(R_{0}\right)$. We have the desired results (i) and (ii).

Case $3\left(\gamma^{*} \leq 0\right)$. The next result considers the case $\gamma^{*} \leq 0$.

Corollary 14. Suppose that $f(t, x)$ satisfies $\left(\mathrm{H}_{1}\right)-\left(\mathrm{H}_{2}\right)$. Furthermore, assume the following.

$\left(\mathrm{H}_{5}\right)$ There exist two positive constants $R>r>0$ such that

$$
\begin{gathered}
R>\Phi_{R 1}+\gamma_{*} \geq r>0, \\
\int_{0}^{1} k(s) g\left(r s^{\alpha-1}\right) d s<+\infty \\
R \geq\left(1+\frac{h(R)}{g(R)}\right) \int_{0}^{1} \frac{(1-s)^{\alpha-1}}{\Gamma(\alpha)\left(1-\beta \eta^{\alpha-1}\right)} k(s) g\left(r s^{\alpha-1}\right) d s,
\end{gathered}
$$

here

$$
\Phi_{R 1}=\int_{0}^{1} \frac{M s(1-s)^{\alpha-1}}{\Gamma(\alpha)\left(1-\beta \eta^{\alpha-1}\right)} \phi_{R}(s) d s .
$$

Then, (28) has at least one positive solution.

Example 15. Suppose that the nonlinearity in (28) be (43) with $k>0, \lambda>0$. If $\gamma^{*} \leq 0, \omega(\lambda)<+\infty$,

$$
\gamma_{*} \geq\left[\frac{\beta_{1} \lambda^{2}}{\beta_{2}^{\lambda}}\right]^{1 /\left(1-\lambda^{2}\right)}\left(1-\frac{1}{\lambda^{2}}\right),
$$

then (28) has at least one positive solution.
Proof. We will apply Corollary 14 . Take $k(t), g(x)$ as the same in the proof of Example 9. Then, $\left(\mathrm{H}_{2}\right)$ is satisfied, and the existence condition $\left(\mathrm{H}_{5}\right)$ is satisfied if we take $R>r>0$ with

$$
\frac{\beta_{1}}{R^{\lambda}}+\gamma_{*} \geq r, \quad R \geq \frac{\beta_{2}}{r^{\lambda}}
$$

and $\omega(\lambda)<+\infty$. If we fix $R=\beta_{2} / r^{\lambda}$, then the first inequality holds if $r$ satisfies

$$
\frac{\beta_{1}}{\beta_{2}^{\lambda}} r^{\lambda^{2}}+\gamma_{*} \geq r
$$

or equivalently

$$
\gamma_{*} \geq l(r):=r-\frac{\beta_{1}}{\beta_{2}^{\lambda}} r^{\lambda^{2}} .
$$

The function $l(r)$ possesses a minimum at

$$
r_{0}:=\left[\frac{\beta_{1}}{\beta_{2}^{\lambda}} \lambda^{2}\right]^{1 /\left(1-\lambda^{2}\right)} .
$$

Taking $r=r_{0}$, then the first inequality in (63) holds if $\gamma_{*} \geq$ $l\left(r_{0}\right)$, which is just condition (62). The second inequality holds directly from the choice of $R$, so it remains to prove that $R=\beta_{2} / r^{\lambda}>r_{0}$. This is easily verified through elementary computations.

Example 16. Let the nonlinearity in (28) be (47) with $k>$ $0, \lambda>0$ and $v \geq 0$. If $\gamma^{*} \leq 0, \omega(\lambda)<+\infty$,

$$
\gamma_{*} \geq m_{0}^{\lambda}\left[\beta_{2}^{1 / \lambda}\left(m_{0}^{1-\lambda^{2}}+\mu m_{0}^{1-\lambda-v-\lambda^{2}}\right)^{1 / \lambda}-\beta_{1}\right],
$$

here $m_{0}$ is the unique solution of the equation

$$
\begin{aligned}
& \beta_{2}^{1 / \lambda}\left(m^{1-\lambda^{2}}+\mu m^{1-\lambda-\nu-\lambda^{2}}\right)^{1 / \lambda-1} \\
& \quad \times\left[m^{1-\lambda^{2}}+\mu(1-\lambda-v) m^{1-\lambda-\nu-\lambda^{2}}\right]=\lambda^{2} \beta_{1} .
\end{aligned}
$$

Then (28) has at least one positive solution.

Proof. We will apply Corollary 14 . To this end, we take $g(x), h(x)$, and $k(t)$ as the same in the proof of Example 10, then $\left(\mathrm{H}_{2}\right)$ is satisfied, and the existence condition $\left(\mathrm{H}_{5}\right)$ is satisfied if we take $R>r>0$ with

$$
\gamma_{*} \geq r-\beta_{1} R^{-\lambda}, \quad\left(1+\mu R^{\lambda+v}\right) \beta_{2} r^{-\lambda} \leq R,
$$

and $\omega(\lambda)<+\infty$. If we fix $R=\left(1+\mu R^{\lambda+v}\right) \beta_{2} r^{-\lambda}$, then the first inequality holds if $R$ satisfies

$$
\gamma_{*} \geq\left[\frac{\left(1+\mu R^{\lambda+v}\right) \beta_{2}}{R}\right]^{1 / \lambda}-\beta_{1} R^{-\lambda} .
$$

Let $m=1 / R$, then

$$
\gamma_{*} \geq m^{\lambda}\left[\beta_{2}^{1 / \lambda}\left(m^{1-\lambda^{2}}+\mu m^{1-\lambda-\nu-\lambda^{2}}\right)^{1 / \lambda}-\beta_{1}\right]=: F(m) .
$$


Then, we have

$$
\begin{aligned}
& F^{\prime}(m) \\
& =\lambda m^{\lambda-1}\left[\left(\beta_{2}\left(m^{1-\lambda^{2}}+\mu m^{1-\lambda-v-\lambda^{2}}\right)\right)^{1 / \lambda}-\beta_{1}\right] \\
& +m^{\lambda}\left[\frac{1}{\lambda}\left(\beta_{2}\left(m^{1-\lambda^{2}}+\mu m^{1-\lambda-v-\lambda^{2}}\right)\right)^{1 / \lambda-1}\right. \\
& \times\left(\left(1-\lambda^{2}\right) \beta_{2} m^{-\lambda^{2}}\right. \\
& \left.\left.+\mu\left(1-\lambda-v-\lambda^{2}\right) \beta_{2} m^{-\lambda-v-\lambda^{2}}\right)\right] \\
& =\frac{1}{\lambda} m^{\lambda-1}\left\{\lambda^{2}\left[\left(\beta_{2}\left(m^{1-\lambda^{2}}+\mu m^{1-\lambda-\nu-\lambda^{2}}\right)\right)^{1 / \lambda}-\beta_{1}\right]\right. \\
& +\beta_{2}^{1 / \lambda}\left[\left(m^{1-\lambda^{2}}+\mu m^{1-\lambda-\nu-\lambda^{2}}\right)^{1 / \lambda-1}\right. \\
& \times\left(\left(1-\lambda^{2}\right) m^{1-\lambda^{2}}\right. \\
& \left.\left.\left.+\mu\left(1-\lambda-v-\lambda^{2}\right) m^{1-\lambda-v-\lambda^{2}}\right)\right]\right\} \\
& =\frac{1}{\lambda} m^{\lambda-1}\left\{\beta_{2}^{1 / \lambda}\left(m^{1-\lambda^{2}}+\mu m^{1-\lambda-v-\lambda^{2}}\right)^{1 / \lambda-1}\right. \\
& \times\left[\lambda^{2}\left(m^{1-\lambda^{2}}+\mu m^{1-\lambda-\nu-\lambda^{2}}\right)\right. \\
& +\left(\left(1-\lambda^{2}\right) m^{1-\lambda^{2}}\right. \\
& \left.\left.\left.+\mu\left(1-\lambda-v-\lambda^{2}\right) m^{1-\lambda-\nu-\lambda^{2}}\right)\right]-\lambda^{2} \beta_{1}\right\} \\
& =\frac{1}{\lambda} m^{\lambda-1}\left\{\beta_{2}^{1 / \lambda}\left(m^{1-\lambda^{2}}+\mu m^{1-\lambda-\nu-\lambda^{2}}\right)^{1 / \lambda-1}\right. \\
& \left.\times\left[m^{1-\lambda^{2}}+\mu(1-\lambda-v) m^{1-\lambda-\nu-\lambda^{2}}\right]-\lambda^{2} \beta_{1}\right\} .
\end{aligned}
$$

Let $F^{\prime}(m)=0$, then we have

$$
\begin{aligned}
\frac{1}{\lambda} m^{\lambda-1}\{ & \beta_{2}^{1 / \lambda}\left(m^{1-\lambda^{2}}+\mu m^{1-\lambda-\nu-\lambda^{2}}\right)^{1 / \lambda-1} \\
& \left.\times\left[m^{1-\lambda^{2}}+\mu(1-\lambda-\nu) m^{1-\lambda-\nu-\lambda^{2}}\right]-\lambda^{2} \beta_{1}\right\}=0 .
\end{aligned}
$$

Now, let us define $\Phi(m)$ by

$$
\begin{aligned}
\Phi(m)=: & \beta_{2}^{1 / \lambda}\left(m^{1-\lambda^{2}}+\mu m^{1-\lambda-\nu-\lambda^{2}}\right)^{1 / \lambda-1} \\
& \times\left[m^{1-\lambda^{2}}+\mu(1-\lambda-v) m^{1-\lambda-\nu-\lambda^{2}}\right] .
\end{aligned}
$$

It is easy to see that $\Phi(m)$ is a nondecreasing function for $m \in[0,+\infty)$ and $\Phi(m) \rightarrow+\infty$, as $m \rightarrow+\infty$. Thus, $\Phi(m)=$ $\lambda^{2} \beta_{1}$ has a unique solution $m_{0}$ such that

$$
\begin{aligned}
& \beta_{2}^{1 / \lambda}(\left.m_{0}^{1-\lambda^{2}}+\mu m_{0}^{1-\lambda-\nu-\lambda^{2}}\right)^{1 / \lambda-1} \\
& \quad \times\left[m_{0}^{1-\lambda^{2}}+\mu(1-\lambda-v) m_{0}^{1-\lambda-\nu-\lambda^{2}}\right]=\lambda^{2} \beta_{1},
\end{aligned}
$$

and $F\left(m_{0}\right)=\inf _{m>0} F(m)$.

So, it remains to prove that $R>r=\left[\left(1+\mu R^{\lambda+v}\right) \beta_{2} / R\right]^{1 / \lambda}$, that is,

$$
m_{0}^{\lambda+1} \beta_{2}^{1 / \lambda}\left(m_{0}^{1-\lambda^{2}}+\mu m_{0}^{1-\lambda-v-\lambda^{2}}\right)^{1 / \lambda}<1
$$

In fact, by (75), we have

$$
\lambda^{2} \beta_{1} \geq\left(\beta_{2} m_{0}^{1-\lambda^{2}}\right)^{1 / \lambda-1}\left(\beta_{2} m_{0}^{1-\lambda^{2}}\right)=\left(\beta_{2} m_{0}^{1-\lambda^{2}}\right)^{1 / \lambda},
$$

that is,

$$
m_{0} \leq\left(\frac{\lambda^{2} \beta_{1}}{\beta_{2}^{1 / \lambda}}\right)^{\lambda /\left(1-\lambda^{2}\right)}
$$

Also we have

$$
\begin{aligned}
\lambda^{2} \beta_{1} \geq & \beta_{2}^{1 / \lambda}\left(m_{0}^{1-\lambda^{2}}+\mu m_{0}^{1-\lambda-v-\lambda^{2}}\right)^{1 / \lambda-1} \\
& \times\left[(1-\lambda-v) m_{0}^{1-\lambda^{2}}+\mu(1-\lambda-v) m_{0}^{1-\lambda-v-\lambda^{2}}\right] \\
= & (1-\lambda-v) \beta_{2}^{1 / \lambda}\left(m_{0}^{1-\lambda^{2}}+\mu m_{0}^{1-\lambda-v-\lambda^{2}}\right)^{1 / \lambda},
\end{aligned}
$$

that is,

$$
\beta_{2}^{1 / \lambda}\left(m_{0}^{1-\lambda^{2}}+\mu m_{0}^{1-\lambda-v-\lambda^{2}}\right)^{1 / \lambda} \leq \frac{\lambda^{2} \beta_{1}}{1-\lambda-v} .
$$

Thus, we have

$$
\begin{gathered}
\left.m_{0}^{\lambda+1} \beta_{2}^{1 / \lambda}\left(m_{0}^{1-\lambda^{2}}+\mu m_{0}^{1-\lambda-v-\lambda^{2}}\right)^{1 / \lambda}\right)^{\lambda} \frac{\lambda^{2} \beta_{1}}{1-\lambda-v} \\
<\left(\left(\frac{\lambda^{2} \beta_{1}}{\beta_{2}^{1 / \lambda}}\right)^{\lambda /\left(1-\lambda^{2}\right)}\right)^{\lambda+1} \\
=\left(\frac{\lambda^{2} \beta_{1}}{\beta_{2}^{1 / \lambda}}\right)^{\lambda /(1-\lambda)} \frac{\lambda^{2} \beta_{1}}{1-\lambda-v} \\
=\lambda^{2 \lambda /(1-\lambda)}\left(\frac{\beta_{1}}{\beta_{2}}\right)^{1 /(1-\lambda)} \frac{\lambda^{2}}{1-\lambda-v}<1
\end{gathered}
$$

since $0<\lambda, \nu<1$, and $1-\lambda-\nu-\lambda^{2}>0$.

We have the desired results. 
Case $4\left(\gamma_{*}<0<\gamma^{*}\right)$.

Example 17. Suppose that the nonlinearity in (28) be (43) with $k>0, \lambda>0$. If $\gamma_{*}<0<\gamma^{*}, \omega(\lambda)<+\infty$,

$$
\gamma_{*} \geq m_{0}^{\lambda}\left[\beta_{2}^{1 / \lambda} m_{0}^{\left(1-\lambda^{2}\right) / \lambda}-\beta_{1}\left(1+\gamma^{*} m_{0}\right)^{-\lambda}\right],
$$

and here $m_{0}$ is the unique solution of the equation

$$
\beta_{2}^{1 / \lambda} m^{\left(1-\lambda^{2}\right) / \lambda}\left(1+\gamma^{*} m\right)^{\lambda+1}=\lambda^{2} \beta_{1}
$$

then (28) has at least one positive solution.

Proof. We will apply Theorem 7. Take $k(t), g(x)$ as the same in the proof of Example 9. Then, $\left(\mathrm{H}_{2}\right)$ is satisfied, and the existence condition $\left(\mathrm{H}_{3}\right)$ is satisfied if we take $R>r>0$ with

$$
\gamma_{*} \geq r-\beta_{1} R^{-\lambda}, \quad \beta_{2} r^{-\lambda}+\gamma^{*} \leq R,
$$

and $\omega(\lambda)<+\infty$. If we fix $R=\beta_{2} / r^{\lambda}+\gamma^{*}$, then the first inequality holds if $R$ satisfies

$$
\gamma_{*} \geq\left(\frac{\beta_{2}}{R-\gamma^{*}}\right)^{1 / \lambda}-\beta_{1} R^{-\lambda} .
$$

Taking $m=1 /\left(R-\gamma^{*}\right)$, then for $m \in(0,+\infty)$, we have

$$
\begin{aligned}
\gamma_{*} & \geq\left(\beta_{2} m\right)^{1 / \lambda}-\frac{\beta_{1} m^{\lambda}}{\left(1+\gamma^{*} m\right)^{\lambda}} \\
& =m^{\lambda}\left[\beta_{2}^{1 / \lambda} m^{\left(1-\lambda^{2}\right) / \lambda}-\beta_{1}\left(1+\gamma^{*} m\right)^{-\lambda}\right]=: F(m) .
\end{aligned}
$$

Then, we have

$$
F^{\prime}(m)
$$

$$
\begin{aligned}
= & \lambda m^{\lambda-1}\left[\beta_{2}^{1 / \lambda} m^{\left(1-\lambda^{2}\right) / \lambda}-\beta_{1}\left(1+\gamma^{*} m\right)^{-\lambda}\right] \\
& +m^{\lambda}\left[\frac{1-\lambda^{2}}{\lambda} \beta_{2}^{1 / \lambda} m^{\left(1-\lambda^{2}\right) / \lambda-1}+\lambda \beta_{1} \gamma^{*}\left(1+\gamma^{*} m\right)^{-\lambda-1}\right] \\
= & \frac{1}{\lambda} m^{\lambda-1}\left\{\lambda^{2}\left[\beta_{2}^{1 / \lambda} m^{\left(1-\lambda^{2}\right) / \lambda}-\beta_{1}\left(1+\gamma^{*} m\right)^{-\lambda}\right]\right. \\
& +\lambda m\left[\frac{1-\lambda^{2}}{\lambda} \beta_{2}^{1 / \lambda} m^{\left(1-\lambda^{2}\right) / \lambda-1}\right. \\
& \left.\left.+\lambda \beta_{1} \gamma^{*}\left(1+\gamma^{*} m\right)^{-\lambda-1}\right]\right\} \\
= & \frac{1}{\lambda} m^{\lambda-1}\left[\beta_{2}^{1 / \lambda} m^{\left(1-\lambda^{2}\right) / \lambda}-\lambda^{2} \beta_{1}\left(1+\gamma^{*} m\right)^{-\lambda-1}\right] \\
= & \frac{1}{\lambda} m^{\lambda-1}\left(1+\gamma^{*} m\right)^{-\lambda-1}\left[\beta_{2}^{1 / \lambda} m^{\left(1-\lambda^{2}\right) / \lambda}\left(1+\gamma^{*} m\right)^{\lambda+1}-\lambda^{2} \beta_{1}\right] .
\end{aligned}
$$

Let $F^{\prime}(m)=0$, then we have

$\frac{1}{\lambda} m^{\lambda-1}\left(1+\gamma^{*} m\right)^{-\lambda-1}\left[\beta_{2}^{1 / \lambda} m^{\left(1-\lambda^{2}\right) / \lambda}\left(1+\gamma^{*} m\right)^{\lambda+1}-\lambda^{2} \beta_{1}\right]=0$.
Now, let us define $\Phi(m)$ by

$$
\Phi(m)=: \beta_{2}^{1 / \lambda} m^{\left(1-\lambda^{2}\right) / \lambda}\left(1+\gamma^{*} m\right)^{\lambda+1} \text {. }
$$

It is easy to see that $\Phi(m)$ is a nondecreasing function for $m \in[0,+\infty)$, and $\Phi(m) \rightarrow+\infty$, as $m \rightarrow+\infty$. Thus, $\Phi(m)=\lambda^{2} \beta_{1}$ has a unique solution $m_{0}$ such that

$$
\beta_{2}^{1 / \lambda} m^{\left(1-\lambda^{2}\right) / \lambda}\left(1+\gamma^{*} m\right)^{\lambda+1}=\lambda^{2} \beta_{1}
$$

and $F\left(m_{0}\right)=\inf _{m>0} F(m)$. is,

So, it remains to prove that $R>r=\left[\beta_{2} /\left(R-\gamma^{*}\right)\right]^{1 / \lambda}$, that

$$
\beta_{2}^{1 / \lambda} m_{0}^{1+1 / \lambda} \leq 1+\gamma^{*} m_{0} .
$$

In fact, by (90), we have

$$
\beta_{2}^{1 / \lambda} m_{0}^{\left(1-\lambda^{2}\right) / \lambda} \leq \lambda^{2} \beta_{1}
$$

that is,

$$
m_{0} \leq\left(\frac{\lambda^{2} \beta_{1}}{\beta_{2}^{1 / \lambda}}\right)^{\lambda /\left(1-\lambda^{2}\right)}
$$

Thus, we have

$$
\begin{aligned}
\beta_{2}^{1 / \lambda} m_{0}^{1+1 / \lambda} & <\beta_{2}^{1 / \lambda}\left(\left(\frac{\lambda^{2} \beta_{1}}{\beta_{2}^{1 / \lambda}}\right)^{\lambda /\left(1-\lambda^{2}\right)}\right)^{(1+\lambda) / \lambda} m_{0}^{1+1 / \lambda} \\
& =\lambda^{2 /(1-\lambda)}\left(\frac{\beta_{1}}{\beta_{2}}\right)^{1 /(1-\lambda)}<1<1+\gamma^{*} m_{0} .
\end{aligned}
$$

The proof is complete.

Example 18. Let the nonlinearity in (28) be (47) with $k>$ $0, \lambda>0$ and $v \geq 0$. If $\gamma_{*}<0<\gamma^{*}, \omega(\lambda)<+\infty$,

$$
\begin{aligned}
\gamma_{*} \geq m_{0}^{\lambda}[ & \beta_{2}^{1 / \lambda}\left(m_{0}^{1-\lambda^{2}}+\mu\left(1+\gamma^{*} m_{0}\right)^{\lambda+v} m_{0}^{1-\lambda-v-\lambda^{2}}\right)^{1 / \lambda} \\
& \left.-\beta_{1}\left(1+\gamma^{*} m_{0}\right)^{-\lambda}\right],
\end{aligned}
$$

here $m_{0}$ is the unique solution of the equation

$$
\begin{aligned}
& \beta_{2}^{1 / \lambda}\left(m^{1-\lambda^{2}}+\mu\left(1+\gamma^{*} m\right)^{\lambda+v} m^{1-\lambda-\nu-\lambda^{2}}\right)^{1 / \lambda-1} \\
& \times\left[m^{1-\lambda^{2}}\left(1+\gamma^{*} m\right)^{\lambda+1}+\mu\left(1-\lambda-\nu+\gamma^{*} m\right) m^{1-\lambda-v-\lambda^{2}}\right. \\
& \left.\quad \times\left(1+\gamma^{*} m\right)^{2 \lambda+v}\right]=\lambda^{2} \beta_{1} .
\end{aligned}
$$

Then (28) has at least one positive solution.

Proof. We will apply Theorem 7. To this end, we take $g(x)$, $h(x)$, and $k(t)$ as the same in the proof of Example 10, then 
$\left(\mathrm{H}_{2}\right)$ is satisfied, and the existence condition $\left(\mathrm{H}_{3}\right)$ is satisfied if we take $R>r>0$ with

$$
\gamma_{*} \geq r-\beta_{1} R^{-\lambda}, \quad\left(1+\mu R^{\lambda+v}\right) \beta_{2} r^{-\lambda}+\gamma^{*} \leq R,
$$

and $\omega(\lambda)<+\infty$. If we fix $R=\left(1+\mu R^{\lambda+v}\right) \beta_{2} r^{-\lambda}+\gamma^{*}$, then the first inequality holds if

$$
\gamma_{*} \geq\left[\frac{\left(1+\mu R^{\lambda+v}\right) \beta_{2}}{R-\gamma^{*}}\right]^{1 / \lambda}-\beta_{1} R^{-\lambda} .
$$

Let $m=1 /\left(R-\gamma^{*}\right)$, then

$$
\begin{aligned}
\gamma_{*} \geq m^{\lambda} & {\left[\beta_{2}^{1 / \lambda}\left(m^{1-\lambda^{2}}+\mu\left(1+\gamma^{*} m\right)^{\lambda+v} m^{1-\lambda-v-\lambda^{2}}\right)^{1 / \lambda}\right.} \\
& \left.-\beta_{1}\left(1+\gamma^{*}\right)^{-\lambda}\right]=: F(m) .
\end{aligned}
$$

Then, we have

$$
F^{\prime}(m)
$$

$$
\begin{aligned}
& =\lambda m^{\lambda-1}\left[\beta_{2}^{1 / \lambda}\left(m^{1-\lambda^{2}}+\mu\left(1+\gamma^{*} m\right)^{\lambda+v} m^{1-\lambda-v-\lambda^{2}}\right)^{1 / \lambda}\right. \\
& \left.-\beta_{1}\left(1+\gamma^{*} m\right)^{-\lambda}\right] \\
& +m^{\lambda}\left[\frac{1}{\lambda} \beta_{2}^{1 / \lambda}\left(m^{1-\lambda^{2}}+\mu\left(1+\gamma^{*} m\right)^{\lambda+v} m^{1-\lambda-v-\lambda^{2}}\right)^{1 / \lambda-1}\right. \\
& \times\left(\left(1-\lambda^{2}\right) m^{-\lambda^{2}}+\mu\left(1+\gamma^{*} m\right)^{\lambda+\nu}\right. \\
& \times\left(1-\lambda-v-\lambda^{2}\right) m^{-\lambda-v-\lambda^{2}} \\
& \left.+\mu(\lambda+\nu)\left(1+\gamma^{*} m\right)^{\lambda+\nu-1} m^{1-\lambda-\nu-\lambda^{2}} \gamma^{*}\right) \\
& \left.+\beta_{1} \lambda\left(1+\gamma^{*} m\right)^{-\lambda-1} \gamma^{*}\right] \\
& =\frac{1}{\lambda} m^{\lambda-1}\left\{\lambda ^ { 2 } \beta _ { 2 } ^ { 1 / \lambda } \left[\left(m^{1-\lambda^{2}}+\mu\left(1+\gamma^{*} m\right)^{\lambda+v} m^{1-\lambda-v-\lambda^{2}}\right)^{1 / \lambda}\right.\right. \\
& -\lambda^{2} \beta_{1}\left(1+\gamma^{*} m\right)^{-\lambda} \\
& +\beta_{2}^{1 / \lambda}\left(m^{1-\lambda^{2}}+\mu\left(1+\gamma^{*} m\right)^{\lambda+v}\right. \\
& \left.\times m^{1-\lambda-v-\lambda^{2}}\right)^{1 / \lambda-1} \\
& \times\left[\left(1-\lambda^{2}\right) m^{1-\lambda^{2}}+\mu\left(1+\gamma^{*} m\right)^{\lambda+v}\right. \\
& \times\left(1-\lambda-v-\lambda^{2}\right) m^{1-\lambda-v-\lambda^{2}} \\
& +\mu(\lambda+v)\left(1+\gamma^{*} m\right)^{\lambda+v-1}
\end{aligned}
$$

$$
\begin{aligned}
& \left.\left.\times m^{1-\lambda-v-\lambda^{2}} m \gamma^{*}\right]\right] \\
& \left.+\lambda^{2} \beta_{1}\left(1+\gamma^{*} m\right)^{-\lambda-1} m \gamma^{*}\right\} \\
& =\frac{1}{\lambda} m^{\lambda-1}\left\{\beta_{2}^{1 / \lambda}\left[\left(m^{1-\lambda^{2}}+\mu\left(1+\gamma^{*} m\right)^{\lambda+v} m^{1-\lambda-\nu-\lambda^{2}}\right)\right]^{1 / \lambda-1}\right. \\
& \times\left[\lambda^{2}\left(m^{1-\lambda^{2}}+\mu\left(1+\gamma^{*} m\right)^{\lambda+v} m^{1-\lambda-v-\lambda^{2}}\right)\right. \\
& +\left(1-\lambda^{2}\right) m^{1-\lambda^{2}} \\
& +\mu\left(1+\gamma^{*} m\right)^{\lambda+v}\left(1-\lambda-\nu-\lambda^{2}\right) m^{1-\lambda-\nu-\lambda^{2}} \\
& \left.+\mu(\lambda+v)\left(1+\gamma^{*} m\right)^{\lambda+v-1} m^{1-\lambda-v-\lambda^{2}} m \gamma^{*}\right] \\
& \left.\times \lambda^{2} \beta_{1}\left(1+\gamma^{*} m\right)^{-\lambda-1} m \gamma^{*}-\lambda^{2} \beta_{1}\left(1+\gamma^{*} m\right)^{-\lambda}\right\} \\
& =\frac{1}{\lambda} m^{\lambda-1}\left(1+\gamma^{*} m\right)^{-\lambda-1} \\
& \times\left\{\beta_{2}^{1 / \lambda}\left(m^{1-\lambda^{2}}+\mu\left(1+\gamma^{*} m\right)^{\lambda+v} m^{1-\lambda-\nu-\lambda^{2}}\right)^{1 / \lambda-1}\right. \\
& \times\left[m^{1-\lambda^{2}}\left(1+\gamma^{*} m\right)^{\lambda+1}+\mu\left(1-\lambda-\nu+\gamma^{*} m\right)\right. \\
& \left.\left.\times m^{1-\lambda-\nu-\lambda^{2}}\left(1+\gamma^{*} m\right)^{2 \lambda+v}\right]-\lambda^{2} \beta_{1}\right\} .
\end{aligned}
$$

Let $F^{\prime}(m)=0$, then we have

$$
\begin{aligned}
& \frac{1}{\lambda} m^{\lambda-1}\left(1+\gamma^{*} m\right)^{-\lambda-1} \\
& \times\left\{\beta_{2}^{1 / \lambda}\left(m^{1-\lambda^{2}}+\mu\left(1+\gamma^{*} m\right)^{\lambda+v} m^{1-\lambda-v-\lambda^{2}}\right)^{1 / \lambda-1}\right. \\
& \times\left[m^{1-\lambda^{2}}\left(1+\gamma^{*} m\right)^{\lambda+1}+\mu\left(1-\lambda-\nu+\gamma^{*} m\right) m^{1-\lambda-v-\lambda^{2}}\right. \\
& \left.\left.\times\left(1+\gamma^{*} m\right)^{2 v+v}\right]-\lambda^{2} \beta_{1}\right\}=0 .
\end{aligned}
$$

Now, let us define $\Phi(m)$ by

$\Phi(m)$

$$
\begin{aligned}
=: & \beta_{2}^{1 / \lambda}\left(m^{1-\lambda^{2}}+\mu\left(1+\gamma^{*} m\right)^{\lambda+v} m^{1-\lambda-v-\lambda^{2}}\right)^{1 / \lambda-1} \\
\times & {\left[m^{1-\lambda^{2}}\left(1+m \gamma^{*} m\right)^{\lambda+1}\right.} \\
& \left.\quad+\mu\left(1-\lambda-v+\gamma^{*} m\right) m^{1-\lambda-v-\lambda^{2}}\left(1+\gamma^{*} m\right)^{2 \lambda+v}\right] .
\end{aligned}
$$


It is easy to see that $\Phi(m)$ is a nondecreasing function for $m \in[0,+\infty)$, and $\Phi(m) \rightarrow+\infty$, as $m \rightarrow+\infty$. Thus, $\Phi(m)=\lambda^{2} \beta_{1}$ has a unique solution $m_{0}$ such that

$$
\begin{aligned}
\beta_{2}^{1 / \lambda}( & \left.m_{0}^{1-\lambda^{2}}+\mu\left(1+\gamma^{*} m\right)^{\lambda+v} m_{0}^{1-\lambda-\nu-\lambda^{2}}\right)^{1 / \lambda-1} \\
\times & {\left[m_{0}^{1-\lambda^{2}}\left(1+\gamma^{*} m\right)^{\lambda+1}\right.} \\
& \left.\quad+\mu\left(1-\lambda-\nu+\gamma^{*} m\right) m_{0}^{1-\lambda-v-\lambda^{2}}\left(1+\gamma^{*} m_{0}\right)^{2 \lambda+v}\right] \\
= & \lambda^{2} \beta_{1},
\end{aligned}
$$

and $F\left(m_{0}\right)=\inf _{m>0} F(m)$.

So, it remains to prove that $R>r=\left[\left(1+\mu R^{\lambda+v}\right) \beta_{2} /(R-\right.$ $\left.\left.\gamma^{*}\right)\right]^{1 / \lambda}$, that is,

$m_{0}^{\lambda+1} \beta_{2}^{1 / \lambda}\left(m_{0}^{1-\lambda^{2}}+\mu\left(1+\gamma^{*} m\right)^{\lambda+v} m_{0}^{1-\lambda-\nu-\lambda^{2}}\right)^{1 / \lambda}<1+\gamma^{*} m_{0}$.

In fact, by (103), we have

$$
\lambda^{2} \beta_{1} \geq\left(\beta_{2} m_{0}^{1-\lambda^{2}}\right)^{1 / \lambda-1}\left(\beta_{2} m_{0}^{1-\lambda^{2}}\right)=\left(\beta_{2} m_{0}^{1-\lambda^{2}}\right)^{1 / \lambda}
$$

that is,

$$
m_{0} \leq\left(\frac{\lambda^{2} \beta_{1}}{\beta_{2}^{1 / \lambda}}\right)^{\lambda /\left(1-\lambda^{2}\right)}
$$

Also we have

$$
\begin{aligned}
& \lambda^{2} \beta_{1} \\
& \geq \beta_{2}^{1 / \lambda}\left(m_{0}^{1-\lambda^{2}}+\mu\left(1+\gamma^{*} m\right)^{\lambda+v} m_{0}^{1-\lambda-v-\lambda^{2}}\right)^{1 / \lambda-1} \\
& \quad \times\left[(1-\lambda-v) m_{0}^{1-\lambda^{2}}\right. \\
& \left.\quad+\mu(1-\lambda-v) m_{0}^{1-\lambda-v-\lambda^{2}}\left(1+\gamma^{*} m\right)^{\lambda+v}\right] \\
& \quad(1-\lambda-v) \beta_{2}^{1 / \lambda}\left(m_{0}^{1-\lambda^{2}}+\mu\left(1+\gamma^{*} m\right)^{\lambda+v} m_{0}^{1-\lambda-v-\lambda^{2}}\right)^{1 / \lambda},
\end{aligned}
$$

that is,

$$
\beta_{2}^{1 / \lambda}\left(m_{0}^{1-\lambda^{2}}+\mu\left(1+\gamma^{*} m\right)^{\lambda+v} m_{0}^{1-\lambda-v-\lambda^{2}}\right)^{1 / \lambda} \leq \frac{\lambda^{2} \beta_{1}}{1-\lambda-v} .
$$

Thus, we have

$$
\begin{gathered}
m_{0}^{\lambda+1} \beta_{2}^{1 / \lambda}\left(m_{0}^{1-\lambda^{2}}+\mu\left(1+\gamma^{*} m\right)^{\lambda+v} m_{0}^{1-\lambda-\nu-\lambda^{2}}\right)^{1 / \lambda} \\
\quad<\left(\left(\frac{\lambda^{2} \beta_{1}}{\beta_{2}^{1 / \lambda}}\right)^{\lambda /\left(1-\lambda^{2}\right)}\right)^{\lambda+1} \frac{\lambda^{2} \beta_{1}}{1-\lambda-v}
\end{gathered}
$$

$$
\begin{aligned}
& =\left(\frac{\lambda^{2} \beta_{1}}{\beta_{2}^{1 / \lambda}}\right)^{\lambda /(1-\lambda)} \frac{\lambda^{2} \beta_{1}}{1-\lambda-v} \\
& =\lambda^{2 \lambda /(1-\lambda)}\left(\frac{\beta_{1}}{\beta_{2}}\right)^{1 /(1-\lambda)} \frac{\lambda^{2}}{1-\lambda-v}<1<1+\gamma^{*} m,
\end{aligned}
$$

since $0<\lambda, v<1$ and $1-\lambda-v-\lambda^{2}>0$.

Thus, the proof is complete.

Remark 19. It is easy to find that analogous results to Examples 10, 13, 16, and 18 for the general equation with the nonlinearity in (28) are

$$
f(t, x)=\frac{b(t)}{x^{\lambda}}+\mu c(t) x^{\nu}+e(t),
$$

with $b, c>0$, but the notation becomes cumbersome. Here we consider the nonlinearity (47) only for the simplicity.

\section{Acknowledgments}

The work was supported by NSFC of China (no. 10971021), the Science and Technology Research Project of Jilin Provincial Department of Education of China (no. 2011176), and the Science and Technology Research Project of Jilin Provincial Department of Education of China (no. 2010391). Supported by Scientific Research Fund of Heilongjiang Provincial Education Department.

\section{References}

[1] A. A. Kilbas, H. M. Srivastava, and J. J. Trujillo, Theory and Applications of Fractional Differential Equations, vol. 204 of North-Holland Mathematics Studies, Elsevier Science B.V., Amsterdam, The Netherlands, 2006.

[2] K. B. Oldham and J. Spanier, The Fractional Calculus, Mathematics in Science and Engineering, vol. 111, Academic Press, New York, NY, USA, 1974.

[3] B. Ross, Ed., Fractional Calculus and Its Applications, Lecture Notes in Mathematics, vol. 457, Springer, Berlin, Germany, 1975.

[4] T. F. Nonnenmacher and R. Metzler, "On the Riemann-Liouville fractional calculus and some recent applications," Fractals, vol. 3, no. 3, pp. 557-566, 1995.

[5] F. B. Tatom, "The relationship between fractional calculus and fractals," Fractals, vol. 3, no. 1, pp. 217-229, 1995.

[6] I. Podlubny, Fractional Differential Equations, vol. 198 of Mathematics in Science and Engineering, Academic Press, San Diego, Calif, USA, 1999.

[7] S. G. Samko, A. A. Kilbas, and O. I. Marichev, Fractional Integrals and Derivatives: Theory and Applications, Gordon and Breach Science, Yverdon, Switzerland, 1993.

[8] R. Magin, X. Feng, and D. Baleanu, "Solving the fractional order Bloch equation," Concepts in Magnetic Resonance Part A, vol. 34, no. 1, pp. 16-23, 2009.

[9] D. Baleanu and J. I. Trujillo, "A new method of finding the fractional Euler-Lagrange and Hamilton equations within Caputo fractional derivatives," Communications in Nonlinear Science and Numerical Simulation, vol. 15, no. 5, pp. 1111-1115, 2010. 
[10] D. Baleanu, K. Diethelm, E. Scalas, and J. J. Trujillo, Fractional Calculus Models and Numerical Methods, vol. 3 of Series on Complexity, Nonlinearity and Chaos, World Scientific, Hackensack, NJ, USA, 2012.

[11] D. Băleanu, O. G. Mustafa, and R. P. Agarwal, "An existence result for a superlinear fractional differential equation," Applied Mathematics Letters, vol. 23, no. 9, pp. 1129-1132, 2010.

[12] D. Băleanu, R. P. Agarwal, O. G. Mustafa, and M. Coşulschi, "Asymptotic integration of some nonlinear differential equations with fractional time derivative," Journal of Physics A, vol. 44, no. 5, Article ID 055203, 9 pages, 2011.

[13] D. Băleanu, O. G. Mustafa, and R. P. Agarwal, "On the solution set for a class of sequential fractional differential equations," Journal of Physics A, vol. 43, no. 38, Article ID 385209, 7 pages, 2010.

[14] Z. Bai, "On positive solutions of a nonlocal fractional boundary value problem," Nonlinear Analysis: Theory, Methods \& Applications, vol. 72, no. 2, pp. 916-924, 2010.

[15] J. Wang, H. Xiang, and Z. Liu, "Positive solution to nonzero boundary values problem for a coupled system of nonlinear fractional differential equations," International Journal of Differential Equations, vol. 2012, Article ID 186928, 12 pages, 2010.

[16] B. Ahmad and J. J. Nieto, "Existence results for a coupled system of nonlinear fractional differential equations with threepoint boundary conditions," Computers \& Mathematics with Applications, vol. 58, no. 9, pp. 1838-1843, 2009.

[17] C. F. Li, X. N. Luo, and Y. Zhou, "Existence of positive solutions of the boundary value problem for nonlinear fractional differential equations," Computers \& Mathematics with Applications, vol. 59, no. 3, pp. 1363-1375, 2010.

[18] X. Xu and X. Fei, “The positive properties of Green's function for three point boundary value problems of nonlinear fractional differential equations and its applications," Communications in Nonlinear Science and Numerical Simulation, vol. 17, no. 4, pp. 1555-1565, 2012. 


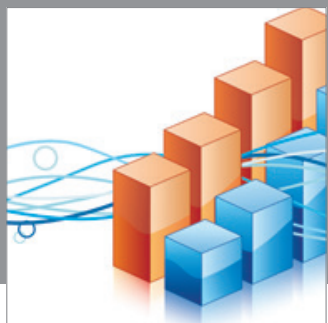

Advances in

Operations Research

mansans

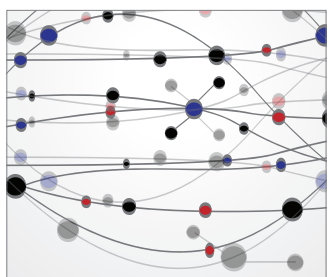

The Scientific World Journal
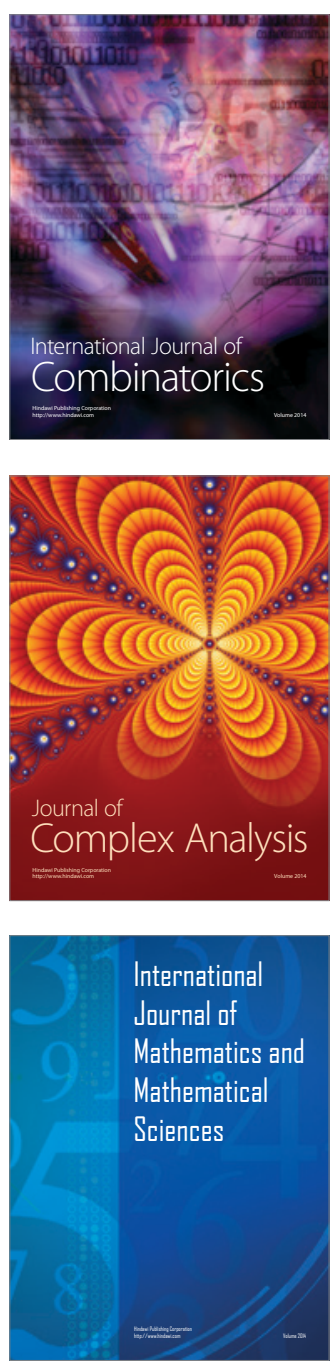
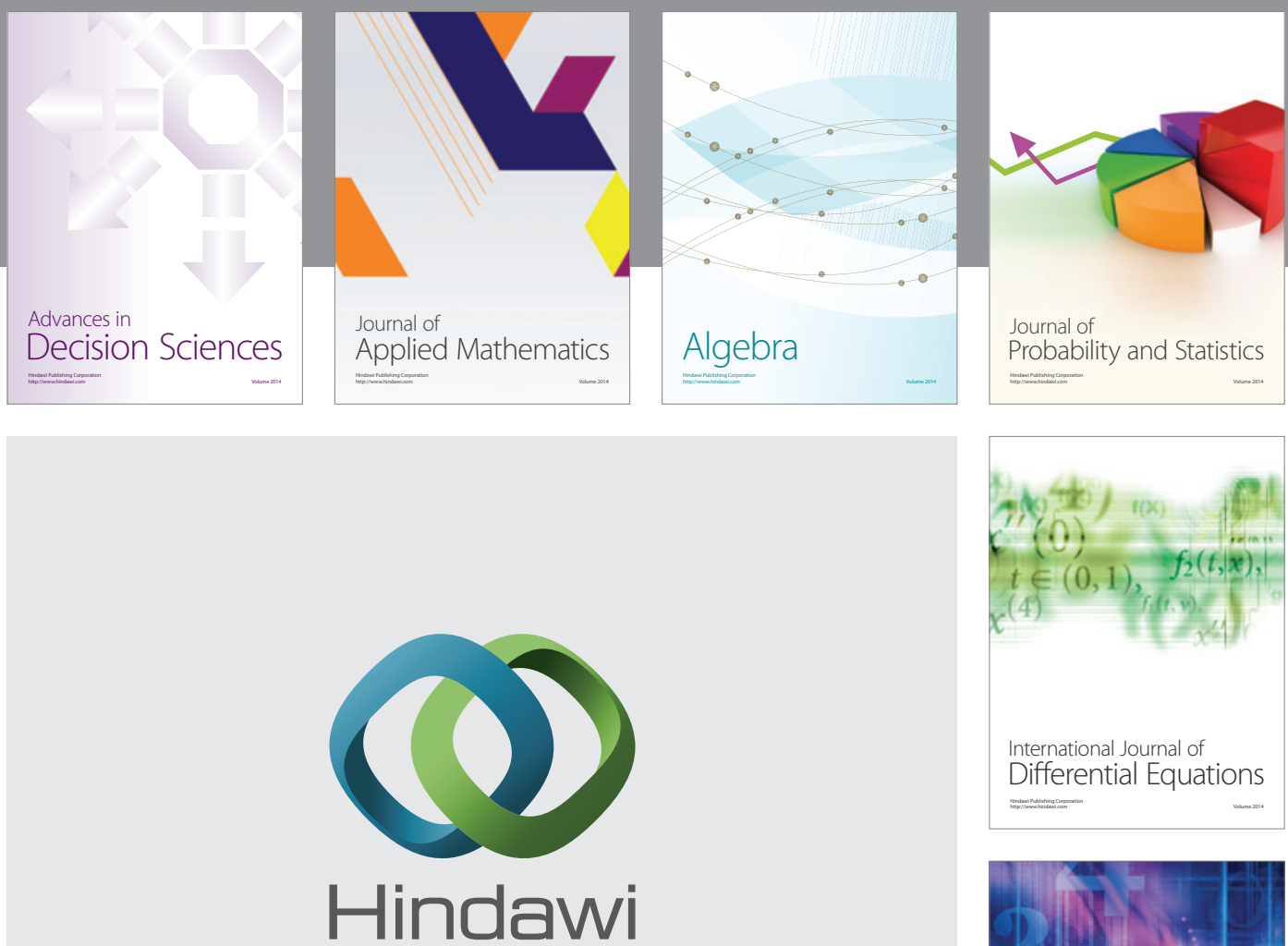

Submit your manuscripts at http://www.hindawi.com
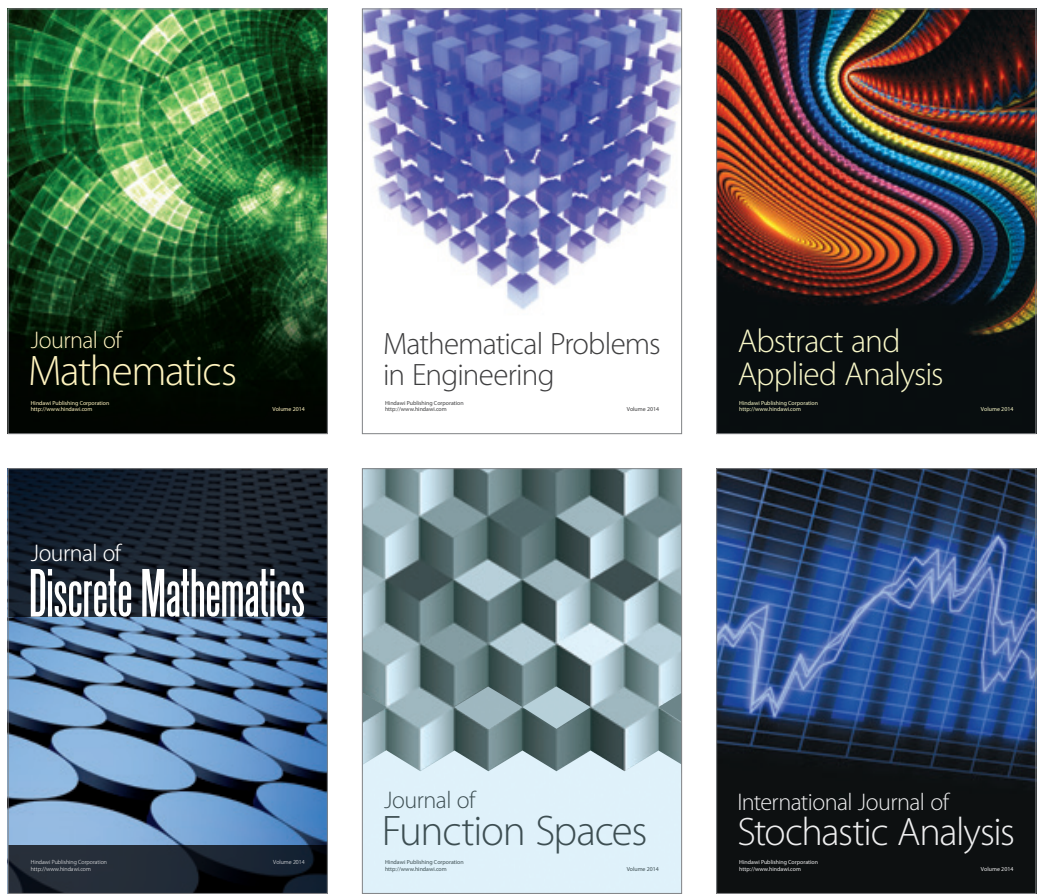

Journal of

Function Spaces

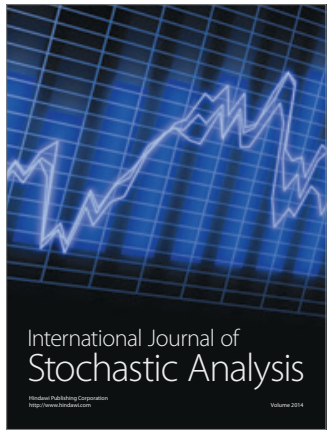

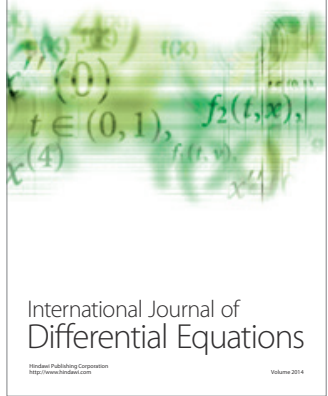
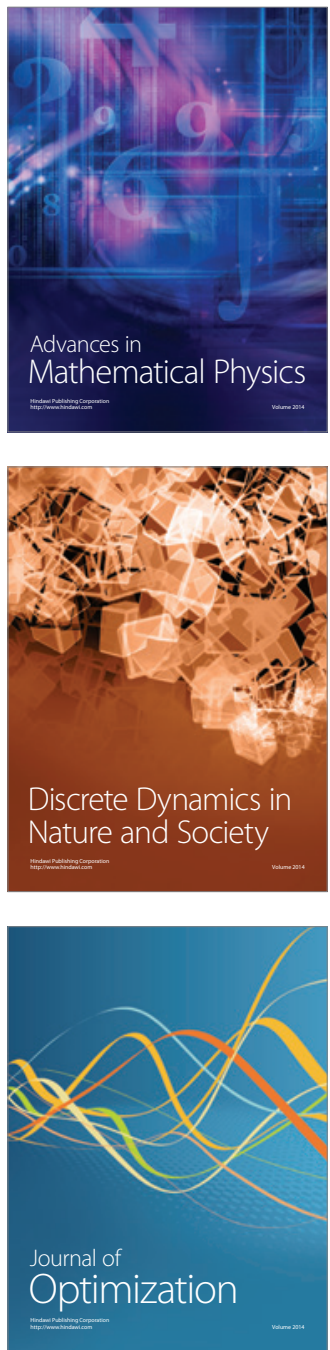